\title{
Demonstrating Doubly-Differential Quadrature Phase Shift Keying in the Optical Domain
}

\author{
Anthony J. Walsh, Haymen Shams, James Mountjoy, Anthony Fagan, Jian Zhao, Liam P. Barry and \\ Andrew D. Ellis
}

\begin{abstract}
We report for the first time the experimental demonstration of doubly-differential quadrature-phase shift keying (DDQPSK) using optical coherent detection. This method is more robust against high frequency offsets (FO) than conventional single differential quadrature phase shift keying (SDQPSK) with offset compensation. DDQPSK is shown to be able to compensate large FOs (up to the baud rate) and has lower computational requirements than other FO compensation methods. DDQPSK is a simple algorithm to implement in a realtime decoder for optical burst switched network scenarios. Simulation results are also provided which show good agreement with experimental results for both SDQPSK and DDQPSK transmission.
\end{abstract}

Index Terms - Phase modulation, burst switching, optical fiber communication.

\section{INTRODUCTION}

$\mathrm{T}$ HERE is a continuing demand for increased bandwidth for optical internet applications [1]. One approach to increasing throughput in the network is to use optical burst switching (OBS)/optical packet switching (OPS) and coherent transmission. OBS and OPS networks have the advantage of removing expensive optical-electronic-optical conversions [2], [3] and also of improving utilization of wavelength division

Manuscript received 07/02/2013.This work was supported by Science Foundation Ireland under grant numbers 09/IN.1/I2653, 06/IN/I969, and 11/SIRG/I2124.

Anthony J. Walsh and Liam P. Barry are affiliated with the Rince Institute, Dublin City University, Glasnevin, Dublin 9, Ireland (phone: 00353 17005883 anthony.walsh66@mail.dcu.ie, liam.barry@dcu.ie).

James Mountjoy and Anthony Fagan are affiliated with the School of Electrical, Electronic and Communications Engineering, University College Dublin, Dublin, Ireland (e-mail james.mountjoy@ieee.org, tony.fagan@ucd.ie).

Jian Zhao and Haymen Shams are affiliated with the Photonics Systems Group, Tyndall National Institute and University College Cork, Cork City, Cork, Ireland (jian.zhao@tyndall.ie, haymen.shams@gmail.com)

Andrew D. Ellis is affiliated with Aston Institute of Photonic Technologies, School of Engineering and Applied Science, Aston University, Aston Triangle, Birmingham B4 7ET, England (email:andrew.ellis@aston.ac.uk).

Anthony J. Walsh was with the Photonics Systems Group, Tyndall National Institute and the Department of Electrical and Electronic Engineering, University College Cork, Cork City, Cork, Ireland.

Andrew D. Ellis was with the Photonics Systems Group, Tyndall National Institute and the Department of Physics, University College Cork, Cork City, Cork, Ireland. multiplexing networks through statistical multiplexing and sub-wavelength grooming [4]. Typically, OBS is implemented using fast switching tunable lasers which often exhibit large time-varying instantaneous frequencies especially directly after a switching event [5]. Coherent transmission requires the use of a coherent optical receiver, a local oscillator (LO) and digital signal processing (DSP) to compensate for system impairments such as frequency offset (FO) [6] and chromatic dispersion [7]. Thus, a robust system for dealing with FO between the tunable laser transmitter and the $\mathrm{LO}$ at the coherent receiver needs to be employed in OBS systems with coherent transmission.

There are many techniques used in DSP for FO compensation (usually combined with phase estimation). One algorithm that allows FO compensation of a modulated carrier is the $\mathrm{m}^{\text {th }}$ power scheme [8]. The received complex signal is raised to the power of $\mathrm{m}$ to give an indication of the carrier phase and estimates the phase drift (and consequently the FO) by comparing the result with the previous estimate (by complex conjugation and multiplication), where $\mathrm{m}$ is the number of constellation points for $\mathrm{M}$-ary phase shift keying. This method can compensate FOs up to a maximum of \pm (Baud Rate) $/(2 \mathrm{~m})$ [6], e.g. $1.25 \mathrm{GHz}$ FO compensation can be achieved for a 10 GBaud quadrature phase shift keying (QPSK) system. Another estimation technique called "predecision-based angle differential estimator" can theoretically compensate for FOs up to \pm (Baud Rate)/2, but this algorithm was demonstrated up to only $5 \mathrm{GHz}$ for $43 \mathrm{Gbit} / \mathrm{s}$ return-tozero QPSK [6]. It should be noted that the FO (between tunable transmitter and LO at the receiver) after the tunable laser switches from its previous wavelength to a new channel, will initially be large, and will then reduce as the laser settles to its target channel. By increasing the range of the FO compensation it becomes possible to successfully transmit data sooner after the switching event and enhance bandwidth utilization in an OBS system.

Doubly differential phase shift keying (DDPSK) [9], previously designed for satellite communications, is theoretically capable of cancelling high FOs in the noise free case. Doubly differential quadrature phase shift keying (DDQPSK) was also simulated in the optical domain in [10] where additional phase noise compensation methods were explored. In this paper, DDQPSK is experimentally demonstrated in the optical domain for the first time, to the 
best of our knowledge. Comparisons between the performance of single differential quadrature phase shift keying (SDQPSK) and DDQPSK in simulation and experiment will show that DDQPSK has a much larger FO range than other FO compensation methods demonstrated in the optical domain, with a FO tolerance range approaching the baud rate. (Note the SDQPSK acronym is used instead of the usual DQPSK for the purpose of clarity.) It has a much lower computational cost than the $\mathrm{m}^{\text {th }}$ power operation which is important for real time implementations. Finally, this DDQPSK demonstration will show that DDQPSK is a suitable candidate format to deal with the large FO values expected in optical burst scenarios and to allow decoding of data sooner after a switching event as the tuning laser will enter DDQPSK's FO tolerance range faster.

\section{THEORY}

DDQPSK works on the premise that FOs can be removed by performing the differential operation twice on the received phase. The encoding and decoding processes for DDQPSK are shown in Figs 1 (a) and 1 (b) [9] respectively.

(a)
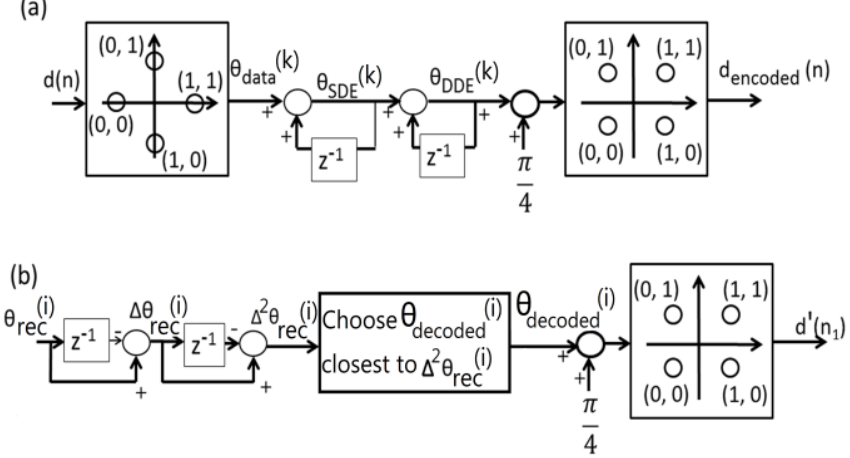

Fig. 1. DDQPSK (a) encoding and (b) decoding [9].

The original data bits, $d(n)$ are translated to phase data, $\theta_{\text {data }}(\mathrm{k})$, using a rotated QPSK Gray coding map, where $\mathrm{n}$ and $\mathrm{k}$ are the indices of the data and phase, respectively. Doubly differential encoding is then applied to the phase data using equation (1) below [9]:

$$
\theta_{D D E}(k)=\theta_{\text {data }}(k)+2 \theta_{D D E}(k-1)-\theta_{D D E}(k-2) \bmod 2 \pi, \mathrm{k} \geq 3
$$

where $\theta_{\mathrm{DDE}}(\mathrm{k})$ is the doubly differential encoded phase. Note in Fig 1 (a) that $\theta_{\mathrm{SDE}}(\mathrm{k})$ is the single differential encoded phase. Then, the encoded phase is rotated by $\pi / 4$ so that it can be mapped using Gray coding back into data bits where the data modulates the transmitter using an IQ modulator (the odd bits were used for I, and even bits for Q). At the receiver, a coherent receiver determines the phase of the beat signal between the LO and the received signal, $\theta_{\text {rec }}(i)$, where $i$ is the index of the received phase. This received phase then undergoes a simple decoding equation given in (2) below:

$$
\Delta^{2} \theta_{\text {rec }}(i)=\theta_{\text {rec }}(i)-2 \theta_{\text {rec }}(i-1)+\theta_{\text {rec }}(i-2) \bmod 2 \pi, \mathrm{i} \geq 3
$$

where $\Delta^{2} \theta_{\text {rec }}(i)$ is the doubly differential phase. The decoded phase, $\theta_{\text {decoded }}(\mathrm{i})$, is chosen as the closest value to the doubly differential phase, where $\theta_{\text {decoded }}(i)$ is an element of $\{0, \pi / 2, \pi$, $3 \pi / 2\}$. Then, the decoded phase is rotated by $\pi / 4$ and is then mapped using Gray coding in order to determine the decoded data, $d^{\prime}\left(n_{1}\right)$, which is expected to be the same as the original data, $d(n)$, where $n_{1}$ is the index of the decoded data.

As shown in [9], in the noise free case, doubly differential decoding completely cancels out the FO and is insensitive to the initial phase of the detected signal at the receiver. If the input phase into equation (2), $\theta_{\text {rec }}(t)$, is composed of phase data $\theta_{\text {encoded }}(t)$, a FO term, $w$, and an initial phase term, $\theta_{0}$, i.e. $\theta_{\text {rec }}(t)=\theta_{\text {encoded }}(t)+w t+\theta_{0}$, then equation (2) becomes:

$$
\begin{aligned}
\Delta^{2} \theta_{\text {rec }}(i)= & {\left[\left(\theta_{\text {encoded }}(t)+w t+\theta_{0}\right)-2\left(\theta_{\text {encoded }}(t-T)+w(t-T)+\theta_{0}\right)\right.} \\
& \left.+\left(\theta_{\text {encoded }}(t-2 T)+w(t-2 T)+\theta_{0}\right)\right] \bmod 2 \pi \\
\Delta^{2} \theta_{\text {rec }}(i)= & {\left[\theta_{\text {encoded }}(t)-2 \theta_{\text {encoded }}(t-T)+\theta_{\text {encoded }}(t-2 T)\right] \bmod 2 \pi(4) }
\end{aligned}
$$

From (4) it can be seen that the doubly differential operation fully removes any FO and initial phase in the noise free case and just leaves the encoded phase terms as required. Hence, doubly differential decoding is completely independent of FO and initial phase offset! In the noise free case, it is clear that the main limiting factor of the FO compensation range is the bandwidth of the electronics. Note that it has been shown that doubly differential binary phase shift keying has some performance degradation as FO increases when additive white Gaussian noise is present [9]. Simulations of DDQPSK show similar degradation but this is insignificant when compared with the effects of the electronic bandwidth. Note that although DDQPSK can deal with very large FOs, very large and fast variations in FO may be problematic. The extent to which this will affect our system is not yet precisely known and we will study the precise performance limits of DDQPSK in the presence of such time-varying FOs in future work.

Shown in Figs 2 and 3 below are the m-th power FO compensation and the mth power phase estimation algorithms respectively used as part of the SDQPSK demodulation [8]. The FO compensation algorithm multiplies the input field by its delayed complex conjugate, gets the $\mathrm{m}$-th power of this, averages this value, divides the phase of the result by 4 , accumulates the resulting values and uses the output of the accumulator to remove the FO from the input field. The m-th power phase estimation averages the $\mathrm{m}$-th power of the input field, averages this value and divides the phase of these averages by 4 , unwraps the result and removes the unwrapped phase from the input field.

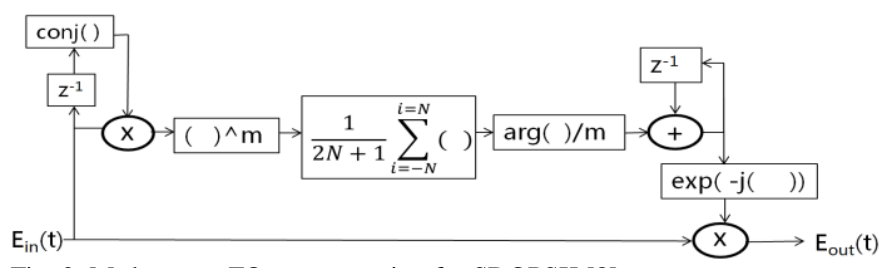

Fig. 2. M-th power FO compensation for SDQPSK [8]. 


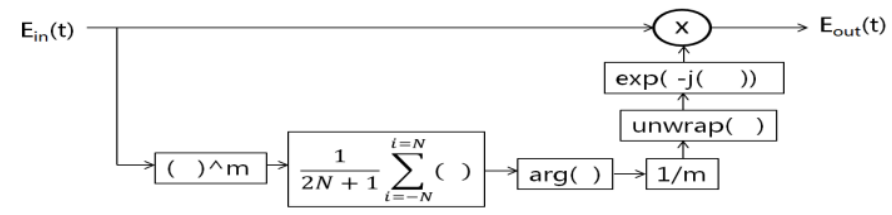

Fig. 3. M-th power phase estimation for SDQPSK [8].

\section{EXPERIMENTAL SETUP}

Simulations and experiments were performed to investigate the performance of DDQPSK and to compare it to SDQPSK using the setup shown in Fig. 4 below. In simulation, fixed frequency lasers were used for the transmitter and LO whilst tunable low linewidth external cavity lasers (ECLs) were used in the experiment. In all cases the data transmitted was first generated in MATLAB commencing with a $2^{7}-1$ pseudorandom bit sequence (with a zero appended to the end as the pattern generator (PG) required patterns of particular lengths). The data was then encoded for either SDQPSK using the encoding scheme described in [8] or DDQPSK [9] transmission. The data signals were then uploaded to the VPI TransmissionMaker V8.7 or PG as appropriate where the data signals were used to drive an IQ modulator to modulate the transmitting laser. The optical signal to noise ratio (OSNR) was adjusted using a variable optical attenuator (VOA), a dual stage optical amplifier (implemented using erbium-doped fiber amplifiers (EDFAs)) and two optical band pass filters (OBPFs) and monitored by an optical spectrum analyzer (OSA) with a resolution bandwidth of $0.14 \mathrm{~nm}$ in the experiment. This bandwidth resolution was corrected to measure the OSNR in $0.1 \mathrm{~nm}$ bandwidth using signal processing. The eye diagram was observed from a $90 \%$ tap using a $50 \mathrm{GHz}$ photodiode (PD) and an oscilloscope which enabled the IQ modulator bias points to be optimized. The other $10 \%$ tap was sent to the polarization-diverse coherent receiver to determine the phase of the received signal. In simulation, the thermal noise of the photodiodes was set to $40 \mathrm{pA} / \sqrt{\mathrm{Hz}}$. A polarization controller (PC) was used to align the polarization of the LO with that of the transmitter. The frequency of the LO was varied between simulations in order to vary the static FO. In the experiment, only a single polarization channel was recorded by a $50 \mathrm{GS} / \mathrm{s}$ real time scope with an analogue bandwidth of $16 \mathrm{GHz}$. This real time scope was triggered by the PG (triggered with the same period as that of the pattern programmed into the PG). The symbol rate was 10 Gbaud. In the experiment, the recorded length was $2 \mu$ s with $2 \times 10^{4}$ symbols $\left(4 \times 10^{4}\right.$ data bits), with the lowest measureable bit error rate (BER) being $2.5 \times 10^{-5}$. The simulations used a similar setup and the OSNR was measured using a resolution bandwidth of $0.1 \mathrm{~nm}$ and 32768 symbols were simulated and a constant 5 samples per symbol was maintained, giving $6.6 \times 10^{4}$ data bits, with the lowest measureable BER being $1.53 \times 10^{-5}$. The electrical bandwidth of the receiver in the simulation was set to be $16 \mathrm{GHz}$ with a Butterworth filter shape to best match experimental conditions.

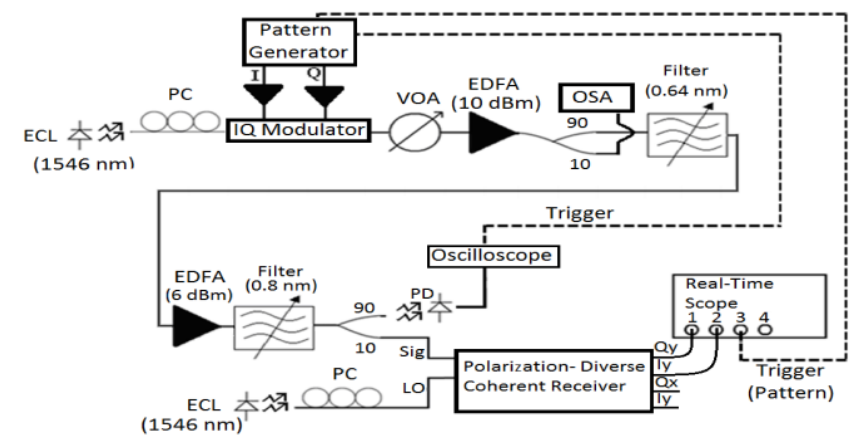

Fig. 4. Schematic diagram of the optical setup used for simulations and experiments.

The DSP was undertaken in MATLAB. For each data set, the electrical signal was down sampled to $10 \mathrm{GS} / \mathrm{s}$. For SDQPSK only, the $\mathrm{m}^{\text {th }}$ power operation was used for FO compensation and phase estimation [8]. For symbol decoding, a single differential operation was used for SDQPSK and a double differential operation was used for DDQPSK (essentially (2) above). Decoding was followed by BER calculation via error counting and sampling phase optimization. This offline DSP process was repeated for different OSNR values and for different FOs.

\section{RESULTS AND DISCUSSION}

Fig. 5 shows BER versus OSNR for different FOs in simulation and experiment for (a) SDQPSK and (b) DDQPSK. It can be seen that simulation and experiment match quite well for SDQPSK and for DDQPSK. Fig. 6 shows the required OSNR for BER of $10^{-3}$ versus FO, for simulation and experiment with SDQPSK and DDQPSK. The required OSNR was determined from the data presented in Fig. 5 with the aid of a linear fit of $\log [-\log [B E R]]$ versus OSNR in $\mathrm{dB}$ (Note that OSNRs less than $39 \mathrm{~dB}$ were used for this fit). The power penalty at zero FO associated with DDQPSK when compared to SDQPSK was approximately $10.5 \mathrm{~dB}$ in simulation and a penalty of $10.6 \mathrm{~dB}$ in experiment (at BER of $10^{-3}$ ). It is clear from both Fig. 5(a) and Fig. 6 that SDQPSK failed when the FO was larger than $1.25 \mathrm{GHz}$ which was expected as discussed in section I. However, DDQPSK could produce BERs of less than $10^{-3}$ for FOs up to $10 \mathrm{GHz}$ in experiment as seen in Figs 5(b) and 6. It can be seen that, from both the simulated and experimental DDQPSK results, the required OSNR for a BER of $10^{-3}$ was less than $30 \mathrm{~dB}$ for FOs of $7 \mathrm{GHz}$ and below. It was found from additional simulations that the performance of DDQPSK at high FOs was mainly affected by the electronic bandwidth of the receiver. Hence, it is envisaged that a larger scope bandwidth would allow for larger FOs.

Another important consideration is that the power penalties seen in simulation and experiment of $10.5 \mathrm{~dB}$ and $10.6 \mathrm{~dB}$ respectively at $0 \mathrm{GHz}$ is much larger than the expected theoretical value of $4.77 \mathrm{~dB}$ (The square modulus of the filter 
in equation (1) is 6, which for an input noise variance $\sigma_{n}^{2}$ will give an output noise variance of $6 \sigma_{n}^{2}$ [11], while for SDQPSK it will be $2 \sigma_{n}^{2}$ [11], meaning the expected penalty in the resulting noise will be $\left.10 * \log 10\left(6 \sigma_{n}^{2} / 2 \sigma_{n}^{2}\right)=4.77 \mathrm{~dB}\right)$. Simulations at $0 \mathrm{GHz}$ FO show that removing the DSP used for SDQPSK does not reduce this discrepancy and, hence, it appears that the DSP is not a factor which contributes to this discrepancy as one might expect.

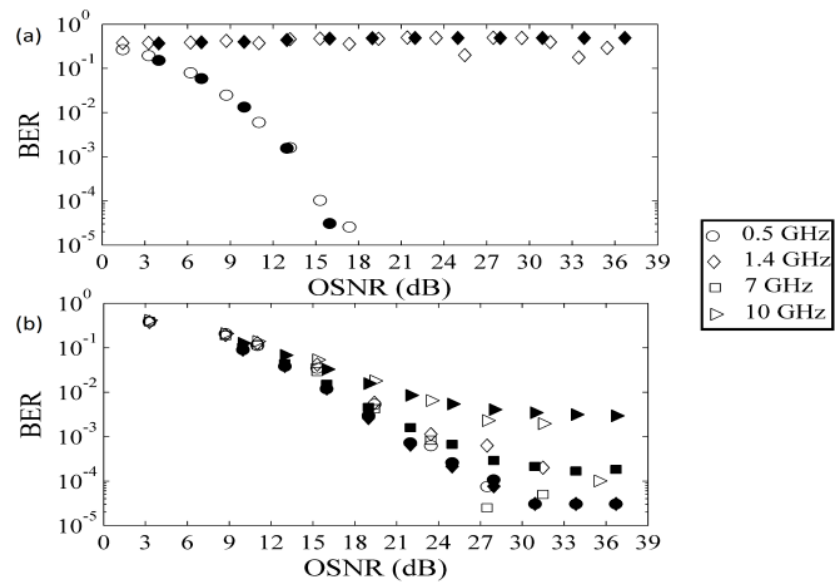

Fig. 5. BER versus OSNR for different FOs for simulated and experimental (a) SDQPSK and (b) DDQPSK. Filled points indicate simulation data and empty points indicate experimental data.

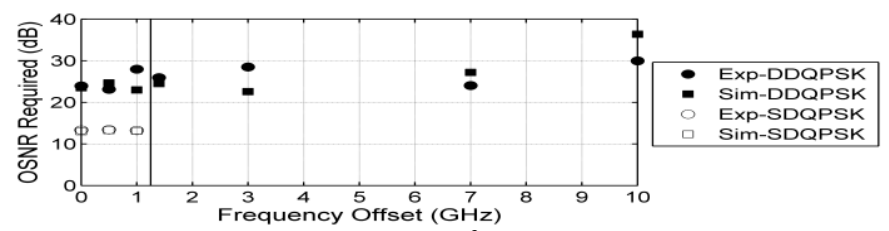

Fig. 6. OSNR required for $\mathrm{BER}=10^{-3}$ versus $\mathrm{FO}$, where the black line indicates the SDQPSK limit. Filled points indicate DDQPSK data and empty points indicate SDQPSK data.

The source of the discrepancy in the simulation is thermal noise in the photodiodes which results in an increased power penalty and the emergence of a noise floor. Simulations confirm that when thermal noise is set to zero at $0 \mathrm{GHz} \mathrm{FO}$, the power penalty for DDQPSK is almost at the theoretical 4.77 $\mathrm{dB}$. As OSNR values become large and ASE noise power becomes lower, the thermal noise will overshadow the ASE noise. This will cause the effective OSNR values to be larger than the measured OSNR values as the ASE noise is decreased. This overestimation of the OSNR values is worse for higher OSNR values since the constant thermal noise will tend to overshadow the decreasing ASE noise. Hence, DDQPSK, which from the theory has higher OSNR requirements than SDQPSK, will have its OSNR requirements overestimated to a greater extent than SDQPSK. This is the source of the power penalty. In addition, the presence of high thermal noise will lead to an upper limit in the effective OSNR values, which leads to an error floor as observed in simulation and experiment. With regard to the experimental results, thermal noise is a possible source for this power penalty and the noise floor. Note that some additional DSP as suggested in [10] can reduce the power penalty associated with DDQPSK and improve performance.

\section{CONCLUSION}

In this paper we have reported the first experimental implementation of DDQPSK in the optical domain. It is clear that DDQPSK has superior performance to SDQPSK with $\mathrm{m}^{\text {th }}$ power operation estimation for FOs in excess of $1.25 \mathrm{GHz}$. This larger frequency range makes DDQPSK more suitable for OBS systems using coherent transmission with fast switching tunable laser transmitters. This is due to the fact that as the transmitting laser's frequency settles to a value close to the LO's frequency, it will be able to reach BERs of $10^{-3}$ faster than other systems. In addition, DDQPSK has lower computation requirements than SDQPSK with $\mathrm{m}^{\text {th }}$ power operation which indicates that DDQPSK would be easier to implement in real-time for OBS network scenarios.

\section{REFERENCES}

[1] D. J. Bishop, et al., "Energy efficient networking: avoiding a future energy crunch," in Proceedings of Military Communications Conference 2010, San Jose, CA, pp. 2047-2049.

[2] Robert Maher, David S. Millar, Seb J. Savory, and Benn C. Thomsen, "Widely tunable burst mode digital coherent receiver with fast reconfiguration time for $112 \mathrm{~Gb} / \mathrm{s}$ DP-QPSK WDM networks", Journal of Lightwave Technology, vol. 30, no 24, pp. 3924-3930, December 2012.

[3] M. Li, et al., "Optical burst-mode coherent receiver with a fast tunable LO for receiving multi-wavelength burst signals," in Proceedings of Optical Fiber Communication Conference 2012, Los Angeles, CA, Paper OTu1G.3.

[4] F. Vacondio, et al., "Real-time implementation of packet-by-packet polarization demultiplexing in a $28 \mathrm{~Gb} / \mathrm{s}$ burst mode coherent receiver," in Proceedings of Optical Fiber Communication Conference 2012, Los Angeles, CA, Paper OM3H.6.

[5] A. K. Mishra, A. D. Ellis, L. P. Barry, and T. Farrell, "Time-resolved linewidth measurements of a wavelength switched SG-DBR laser for optical packet switched networks," in Proceedings of Optical Fiber Communication Conference 2008, San Diego, CA, Paper OTuC4.

[6] L. Li, et al., "Wide-range, accurate and simple digital frequency offset compensator for optical coherent receivers," in Proceedings of Optical Fiber Communication Conference 2008, San Diego, CA, Paper OWT4.

[7] J. Zhao and H. Shams, "Fast dispersion estimation in coherent optical 16QAM fast OFDM systems", Optics Express, vol. 21, no. 2, pp. 2500 2505, January 2013.

[8] X. Liu, S. Chandrasekhar, and A. Leven, "Self-coherent optical transport systems," in Optical Fiber Telecommunications V B: Systems and Networks, I. P. Kaminow, T. Li, and A. E. Willner, (Academic Press, Oxford 2008) pp. 131-178.

[9] D. K. van Alphen and W. C. Lindsey, "Higher-order differential phase shift keyed modulation," IEEE Transactions on Communications, vol. 42, no. 2/3/4, pp. 440-448, February/March/ April 1994.

[10] M. Nazarathy, A. Gorshtein, and D. Sadot, "Doubly-differential coherent 100G transmission: multi-symbol decision-directed carrier phase estimation with intradyne frequency offset cancellation," in Proceedings of Signal Processing in Photonic Communications 2010, Karlsruhe, Germany, Paper SPWB4.

[11] R. G. Albano, Luisa Franchina, and Savvas A. Kosmopoulos, "Bit Error Performance Evaluation of Double-Differential QPSK in Faded Channels Characterized by Gaussian Plus Impulsive Noise and Doppler Effects", in IEEE Transactions on Vehicular Technology, vol. 49, no. 1, pp. 148-158, January 2000. 